\title{
4 Age Dynamics and Intergender Differeces in the Level of Coordination Abilities
}

In sport games, and particularly in volleyball, the importance of technical and coordination performance factors has recently increased, since their level significantly influence the tempo, quality and stability of the acquisition of sport skills. In this way they facilitate the course of movement in the improvement and stabilization phases, determine the degree of utilization of the conditioned potential and allow for flexible adaptation of the movement to constantly varying conditions.

Based on several research works of mostly German sport scientists we can accept the fact that development of coordination abilities has a different course than that of the conditioning performance potentials (Hirtz, 1985; Juřinová, 1982; Ljach, 1988; Hartmann, 1992; Šimonek, 1993, 1998; Šimonek, et al., 1997, 1999; and others). Even if these authors applied various research methods, they basically agree that the most optimum interval for the development of coordination abilities can be considered between 7 and 12 years. This is the period of an intensive development of motor centres in the brain. At the age of 8 , the development of the brain and head of children is already $90-95 \%$ of the maximum value (Weineck, 1996). "Fair reaction speed, courage, favourable somatic prerequisites, sufficient level of inevitable motor abilities, as well as high level of excitability and plasticity of the nerve system create favourable conditions for acquisition of the most difficult coordination movements" (Winter, 1975), (Fig. 4.4).

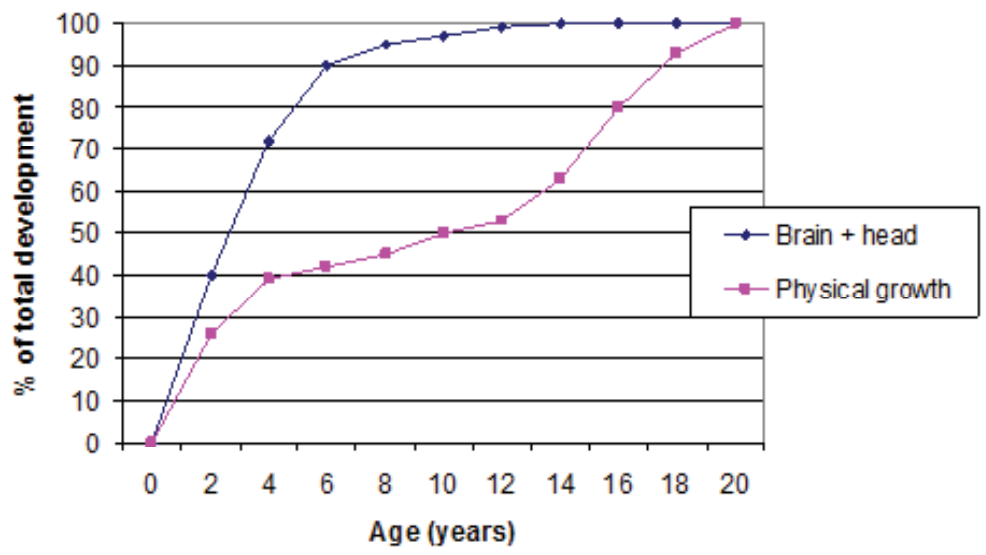

Fig. 4.4: Development of brain, head and overall somatic growth until adulthood (Weineck, 1996) Explanations: Blue line $=$ development of brain and head, red line = overall somatic development 
Based on the research results as well as practical experience we can assume that between the ages of 7-12 years children can perform almost all movements (even the most complex), unless an extreme range of movements, considerable power, speed or endurance are required. That is why we can start teaching them even very difficult, complex motor skills (technique), which occur in sport games for example, lay-up in basketball, jump-up and spike in volleyball, timed pass in ice-hockey or soccer.

Israel (1977) emphasised the necessity to start methodical development during the pre-school years. Children upon entering school are able to successfully rate their efforts, to conform them to a certain rhythm, to slow them down.

The most intense phase of development is during the ages of 7-10 years while the overall development is almost finished in the first half of the school age. After the age of 12, there follows an interval of slower developmental dynamism and partial stagnation. This is conditioned both biologically and socially. In the initial school ages, in spite of the fact that a child shifts from the period of "free spontaneous movement" into the one of calmer, sedentary way of life, the child is developing under progressive conditions and age-specific changes in relation to the nervous system. Morphological development of the nervous system is almost completely closed until sexual adulthood. The kinesthetic-motoric analyser matures very early.

"Differentiation of spatial parameters, perception of time, maximum movement frequency of legs and fine motoricity of hands reach almost $75 \%$ of the overall increase for the whole school period already between 10th and 11th years of age. There comes also to a clear developmental shift in simple motor reaction to optical and acoustic signals similarly as upon distributing the attention. At the ages of compulsory school attendance there comes to an even development in space orientation ability, optical - spatial perception, differentiation of temporal parameters (about $75 \%$ of the total increase)" (Hirtz, 1985).

P.E. at school offers only a limited motor activity of children and youth, which causes delayed development in the growth of coordination abilities. Further development of substantially matured function of movement control is possible only through intensive stimulation of motor activity in children and youth.

Another reason can be low coordination demands in the pre-school age and younger school age (Frömel, 1982). Research by Zimmermann and Nicklisch (1981); Hirtz (1985); Brandt (1985); Raczek (1990); Szczepanik and Szopa (1993); Zat'ková (1993); Šimonek (1994) and others suggest that by means of a long-term, systematic development of coordination abilities it is possible to reach positive changes in both sexes (by up to $15-20 \%$ for 12 months). It has been found that children involved in spontaneous sport activity in their spare time, do not experience stagnation of coordination development after their 12th year .

Problems of intergender differences in the level of coordination abilities have not yet been completely solved. Results of partial research are often contradictory and depend on the methodolgy used. Some authors (Raczek \& Mynarski, 1992) think there is a differentiated development between boys and girls only after the commencement 
of puberty, while boys and girls by turns gain superiority in the quality of individual coordination abilities. Other authors (Hirtz, 1985; Ljach, 1990) suggest that although psychophysical functions show only minimal differences, in coordination abilities these were typical mainly after the age of 14 . While it is possible to assume that the coordination maturity in girls occurs approx. 1 - 1.5 years earlier than in boys, the phases of stagnation are even more expressly marked.

Górska and Gierat (1995) concluded that no statistical differences in the level of coordination abilities were found in 13 year olds. Girls reached similar results as boys in the development of psychological - regulatory-controlling - processes, which determine the level of coordination abilities.

Šimonek etal.(2000) havenot found (with someindividual exceptions) statistically significant intergender differences between $6-22$ year-olds coming from the regions of Nitra, Brno and Szeged (Figures 1-7, 22-25 enclosed). These researchers also found intergender differences only in space-orientation abilities, which suggests that brain structure differs in both sexes which is then manifested in functional differences relating to spatial visualization, perception of spatial parameters of movement and their changes. Males seem better equipped at solving spatial tasks while females fare better in precisely executing any motor tasks.

According to Kasa and Šimonek (1999) both men and women are similar in terms of coordination abilities which is why it is suggested that the development of coordination abilities during initital stages of sport prepartion in boys and girls should not substantially differ.

Lifelong courses of change the level of coordination abilities have an individual character in each individual. This is supported by longitudinal research carried out by Starosta and Hirtz (1985), who included in experimental group all children, not only those motorically talented. The authors discovered that a girl who grew $18 \mathrm{~cm}$ over 2 consecutive years showed decreased levels of coordination abilities (a drop of 17 points) which consequently negatively influenced the process of acquisition of acquiring technique in sport training. Conversely, a girl who acquired early development of coordination abilities, showed that the growth spurt did not effect skill acquisition for a long time, and the level of coordination abilities started to increase again after a short period of stagnation. These experiences suggest that at an early development of coordination abilities and individual differentiation of requirements we can reach positive improvement of quality of the sport preparation of children and youth.

\subsection{Peculiarities in the Development of Coordination Abilities at Ages 6-9 Years}

The age of 6-9 years is an especially favourable period in the development of coordination abilities (Graphs 22 - 25). Girls during this age increase $28.6-92.3 \%$ 
(on average 62.3\%) and boys 22.5 - $80.0 \%$ (on average $56.2 \%$ ) in indicators of coordination abilities (Ljach, 1989). That means that it is easily possible to achieve the development of these abilities in younger school ages, when there is an accelerated improvement of individual coordination abilities. If we neglect this period of accentuated external effect, we cannot expect a larger developmental effect in the following age category.

From the point of view of sequentiality influencing individual coordination abilities we can determine according to Hirtz (1985) the following order of influencing coordination abilities: at the age of 7-9 years-mainly kinesthetic-differentiation and reaction speed; at the age of 9-11 years - reaction and rhythmic abilities; and the end of this period-balance (especially dynamic balance). During the ages of 7-11 years there is a substantial increase in fine motor skill, accuracy and rhythmic perception based on the high tempo of motor learning.

Based on our own research with children aged 5-6 through 10 years we recommend to develop coordination abilities in the pre-school age, as well as in the younger school age with an increased stress on the development of courage through increasing the risk-taking in a given motor task, and increased changing of conditions of exercises and motor rhythm.

It is necessary that teachers and trainers already in creches and primary schools regularly monitor the level of coordination abilities of children and assess changes over time. Knowing the tempo of motor learning as well as the level of motor abilities will enable teachers to suitably select and dose exercises and games with the aim of improving motor skills in children.

Through accented and variable training it is possible to reach remarkable changes in the course of development of coordination prerequisites for sport performance. This has been highlighted by several longitudinal experiments (Hirtz, 1985), cross-sectional studies (Ljach, 1989; Belej, Starosta, \& Bajdziňski et al., 1994; Šimonek, 1997, 1999, and others), and also pedagogical experiments (Raczek, 1990; Zat'ková, 1993; Szczepanik, 1993; Šimonek, 1994). At the end of the experiment, 11 year old children reached the performance of 15-16-year-olds. Particularly large increases were recorded in reaction speed, balance and kinesthetic-differentiation ability, while in the case of movements requiring fast acceleration and agility, the results were observed only after the first or even second years of experimentation. Rhythmic ability is improved depending on the age along with the maturing of the nervous system in children. No statistically significant inter-gender differences were found between boys and girls (Šimonek, 1999).

School sport has a positive influence on gross differentiation of muscular effort, fine proprioceptive perceptions should be developed by means of special means. Special groups of selected athletes show better ability of rhythmic perception and rhythmic performance on average than the common population. For example, tennis players and basketball players react better to acoustic stimuli. Young athletes, who train at least 4 times a week, show much higher indicators of this ability. 
Table 4.9a: Percentage of individual performances of boys in the test "distance estimation"

\begin{tabular}{lllll}
\hline Age & \multicolumn{2}{l}{ \% share } & 1st attempt & \multicolumn{2}{l}{ \% share 2nd atempt } \\
\hline \multirow{2}{*}{ 6-year-old } & 12.5 & 6.2 & 12.5 & 6.3 \\
\cline { 2 - 5 } & 40 & 41.3 & 33.7 & 47.5 \\
\hline \multirow{2}{*}{ 7-year-old } & 25 & 10 & 15 & 10 \\
\cline { 2 - 5 } & 35 & 30 & 30 & 45 \\
\hline \multirow{2}{*}{ 8-year-old } & 16.7 & 27.8 & 19.4 & 14 \\
\hline \multirow{2}{*}{ 9-ear-old } & 19.4 & 36.1 & 33.3 & 33.3 \\
\hline \multirow{2}{*}{ 10-year-old } & 3.3 & 6.7 & 3.3 & 16.7 \\
\cline { 2 - 5 } & 23.3 & 66.7 & 35 & 45 \\
\hline
\end{tabular}

Explanations: Children had to drop a small cube as close to the centre of a square on the floor as possible. We divided the square into 4 smaller squares. Each square was marked with two symbols. Symbol (-) in the first position meant that the child placed the cube to the left from the centre, while symbol (+) in the first position meant that the cube was placed to the right. When symbol (-) was used in the second position it meant that the cube was placed nearer from the start position than the centre of the cube, while symbol (+) in the second position meant that the cube was placed too far (behind the centre).

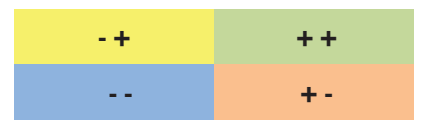

When observing the level of distance estimation and motor memory we found out that with the growing number of attempts the inaccuracy of performing the motor task increased. We carried out testing, where children were asked to drop a small cube to the centre of a square $(1 \mathrm{~m} \times 1 \mathrm{~m})$ at the distance of $10 \mathrm{~m}$. They performed the task with their eyes closed. Based on the visual control after each attempt the movement performance was adjusted. In both sexes the ability to estimate the distance (in both protocols - with and without executing a $360^{\circ}$ degree turn) is improving with the growing age. It is a paradox that in the test of distance estimation (protocol with a turn) the tested persons reached mostly better results than in the test protocol without a turn. We assume that under the influence of motor experience reached through individual attempts there comes to the improvement of motor realization of the set task, in spite of impairing the orientation by performing the turn (360 degrees) (Tab.4.9a, 4.9b). 
Table 4.9b: Percentage of individual performances of girls in the test "distance estimation"

\begin{tabular}{lllll}
\hline Age & \multicolumn{2}{l}{ \% share } & 1st attempt & \multicolumn{2}{l}{ \% share 2nd atempt } \\
\hline \multirow{2}{*}{ 6-year-old } & 40 & 6.5 & 31.2 & 3.2 \\
\cline { 2 - 5 } & 17.5 & 36 & 36.2 & 29.4 \\
\hline \multirow{2}{*}{ 7-year-old } & 4.5 & 4.5 & 44.5 & 9 \\
\hline \multirow{2}{*}{ 8-year-old } & 54.5 & 36.5 & 36.5 & 50 \\
\hline \multirow{2}{*}{ 9-ear-old } & 6.3 & 15.6 & 28.1 & 12.5 \\
\hline & 37.5 & 40.6 & 40.6 & 18.8 \\
\hline \multirow{2}{*}{ 10-year-old } & 5.9 & 11.8 & 11.7 & 17.7 \\
\hline & 5.5 & 47 & 29.4 & 41.2 \\
\hline
\end{tabular}

Gerhát, Kollárovits and Teplická (1995) found the largest influence of motor learning on perception and differentiation of the spatial parameter. Ability to differentiate space was approximately identical in both sexes and the largest intraindividual differences were recorded only in the pre-school age, when probably the development of functional prerequisites of the organism of children had been concluded. Kollárovits and Gerhát (1993) propose that due to the unreliability of participants' performances in various age categories the level of certain coordination abilities is unstable.

Šimonek et al. (1999) observed a tendency to deviate when bringing an object to a target visual control is control is deprived after prior accurate distance estimations. The authors found out that $75 \%$ of participants brought the object shorter and more to the right (values minus-minus and plus-minus), which can be attributed to the influence of fear and uncertainty while performing the task. The deviation to the right side may result from the stronger right leg in right-legged pupils, who form the majority of the population.

\subsection{Peculiarities in the Development of Coordination Abilities at the Age of 10-17 Years}

The age period between 10 and 17 years (period of middle and older school ages) can be devided into three shorter periods which differ depending on gender (Graphs 22-25). At the beginning of this period there is no natural growth in the level of 
coordination abilities. However, in girls approximately at the age of 11-12 years puberty starts, which is marked by the negative influence of morphological indicators on the level of coordination. (See enclosed graphs). Puberty disturbs mostly balance and kinesthetic - differentiation abilities.

Boys enter puberty by 1-1.5 years later, thus prolonging the period of coordination disruption until the age of 14-16 years (Riegrová \& Ulbrichová, 1998). After this period, in boys and girls we can record favourable increases in the level of certain coordination abilities, mostly space-orientation ability, balance and reaction speed (Šimonek et al., 1997; Hirtz, 1985).

Age dynamics of coordination abilities in the interval between 10-17 years has similar characteristics both in boys and girls and records an increasing trend with the exception of rhythmic ability (boys) and kinesthetic-differentiation ability of arms (boys - decrease, girls-stagnation). Stagnation and decreasing level of rhythmic ability in boys can be explained by the fact that boys generally do not engage in rhythmical exercises (rope skipping and the like) by the second grade of elementary school while girls continue in intentional development of rhythmic ability within the lessons focused on rhythmic and aerobic gymnastics. This depends on specific countries curriculum policy.

Worsened performance in boys when compared with girls in the test of kinestheticdifferentiation ability of arms ("Sitting target throw") can be caused by the growth in power in boys after the age of 11, which, however, misrepresents the performance in the precision throw (Šimonek et al., 1997).

Regarding complexity of the structure of coordination abilities and difficulty of their diagnosing, it is not so easy to characterize their developmental curves. Results in tests do not depend only on the level of the particular coordination ability but also on other motor abilities, which impact the resulting performance (e.g. performance in the test of space-orientation (Hirtz, 1985) does not depend only on the spaceorientation factor but also other factors like reaction speed, acceleration speed, etc.). It is difficult then to determine to what degree the result in the test reflects the level of the coordination ability we intend to measure, and to what degree the level of other participating motor abilities.

Factors participating in the resulting performance are represented by a different measure which depends also on sensitive periods of their development. Spaceorientation ability tested by a "Shuttle run" is an example. The level of this ability is increased in the pre-school age and in puberty. Girls reach their maximum at the age of 13 and boys at the age of 17 . Then follows a gradual regression. Intergender differences occur only in the period of late puberty.

Movement accuracy depends mainly on the level of proprioception and the structure of joints. The largest increase in the level of this ability is reached by children aged 7-8 years. Another improvement comes between the 10th and 13th year and according to authors Riegerová and Ulbrychová (1998) it is related to the period of growth acceleration. Movement accuracy in upper extremities tested by a "Target 
throw" increases during childhood and adolescence, while the first peak in girls is reached at the age of 17 and in boys even much later. Regression in boys is even much more intense than in girls.

Static balance depends on the nervous system, particularly the vestibular system. The highest level is reached after year 17, at first in women and later in men. A massive growth in this coordination ability is between years 11 and 12 and later in boys after the age of 14, in girls a year later. Between years 11 and 13 in boys and 12 and 15 in girls there comes to stagnation and even decrease. Static balance in the pre-school age is better in girls, however, later boys take the lead.

Results of observation of dynamic balance show that this motor ability is provided by the level of nervo-muscular coordination and development of the nervous system (vestibular system). Large increase in the level of dynamic balance are recorded in the pre-school age, whereas during puberty we witness large oscillations with a marked regression in girls between the age of 12 and 14, in boys between 14 and 16. These intense oscillations are probably connected with the changes of the overall proportionality and growth acceleration, which are the causes of the change of the position of body centre of gravity along with the disruption in static balance.

Generally speaking, the most favourable periods are in first five years of school attendance and then after the end of puberty. Children obtain rudiments of motor coordination in the first grade of elementary school, on which they can build-up their sport mastery during their motor improvement programmes. However, one should not forget about the parallel improvement of coordination abilities and acquisition of motor skills, mainly later in the middle school age period.

Based on his research Hirtz (1985) specified that kinesthetic differentiation (function of motor analysers) develops first, a bit later reaction speed and relatively late the ability of space orientation (especially functions of static-dynamic and optic analysers). The development partially shows insignificant intergender differences: boys at first lag behind and later, as a result of being involved in sports, they develop their coordination abilities after the age of 13, which can be attributed also to the development of speed and speed-strength abilities at the sensitive period of 12-14 years. These facts suggest that coordination maturity precedes the sexual one.

One of the very few intergender differences, which coaches should take into consideration while planning the conditioning-coordination preparation, is the fact that coordination development is shifted forward by 1 to 1.5 years in girls and therefore the most suitable periods for the development of coordination abilities will also be prolonged. 


\subsection{Age Peculiarities in the Development of Coordination Abilities at the Age of 18-22 Years}

No researchers have observed the level of coordination abilities in the age category of 18 and over so far. According to our research (Šimonek et al., 2000) we can list at least some preliminary facts (Graphs $22-25$ ):

1. After the age of 18 , coordination abilities are stable with only minimal deviations, in dynamic balance where development continues until a peak at the age of 22 years (Riegerová \& Ulbrichová, 1998) and in space-orientation which shows a decrease.

2. After a transitory decrease in the level of coordination abilities at the beginning of puberty and a consecutive second favourable period (showing a positive influence), there are no marked shifts forward in the level of these motor prerequisites.

3. We have not found any significant gender differences in levels of individual coordination abilities in this age.

4. Even in the the period of junior and adult ages it is possible to reach certain positive changes in the level of coordination abilities, however, it requires a focused and long-term effort with specific focus of the sport preparation, especially in the preparatory and competitive periods. 